\section{P138 PROGRESS OF COVID-19 SURVIVORS AND THE IMPACT OF THE INFECTION ON THEIR ABILITY TO RETURN TO WORK}

${ }^{1} \mathrm{D}$ Siaw Hui Min, ${ }^{1} \mathrm{M}$ Ahmed, 'S Mason, ' A Keegan, ${ }^{1} \mathrm{P}$ Vetrivel, ${ }^{1} \mathrm{P}$ Verma, ${ }^{1} \mathrm{R}$ Huang, ${ }^{1} \mathrm{Y}$ Ko, ${ }^{1} \mathrm{C}$ Apthorp, ${ }^{1} \mathrm{~A}$ Twabie, ${ }^{1} \mathrm{C}$ Moret, ${ }^{1,2} \mathrm{H}$ Badri, ${ }^{1} \mathrm{JL}$ Hoyle. ${ }^{1}$ North Manchester General Hospital, Manchester University Foundation NHS Trust, Manchester, UK; ${ }^{2}$ Division of Infection, Immunity and Respiratory Medicine, The University of Manchester, Manchester UK

\subsection{6/thorax-2021-BTSabstracts.247}

Background Prior studies have suggested that certain occupations, such as health and social care are associated with a higher risk of severe COVID-19 infections and increased morbidity. Recent data suggests that even patients with mild COVID-19 infection can have persistent symptoms several months after the acute illness has resolved. We sought to assess rates of return-to-work and the impact of severity of COVID-19 infections and morbidity.

Methods Patients discharged with positive COVID-PCR swab between 06/04/2020 and 16/04/2021 from our district general hospital were entered into a registry and systematically followed up by telephone virtual clinic at 4-6 weeks post discharge. Baseline characteristics, smoking status, COVID-19 severity (severe COVID defined as the need for intubation or CPAP), comorbidities and occupation were collected. In the present study only patients aged 18-65 years, and those with complete baseline data were included. At follow up return to work status and morbidity were recorded (severe morbidity defined as the presence of 3 or more symptoms of breathlessness, fatigue, psychological symptoms and sleep disturbance). Interactions between return-to-work status (back at work $\mathrm{v}$ not back at work) and severe COVID, and severe morbidity were assessed with Chi-Squared Test. Statistical significance $\mathrm{p}<0.05$.

Results In total 254 patients were included (80\% Caucasian, median age 56 [lower quartile 47 to upper quartile 63] years, $48 \%$ female, $41 \%$ current smokers were included. Of these 138 (54\%) patients were employed (table 1). In the employed cohort $11 \%$ of patients had severe COVID. Follow up data was complete in 30 patients, of whom 58\% had returned to work and 50\% had severe morbidity. Morbidity at follow up included sleep disturbance (78\%), sleep (72\%), fatigue $(53 \%)$ and shortness of breath (42\%). There were no significant interactions between return-to-work status and COVID severity at presentation $(p>0.05)$ and between return-to-work status and severe morbidity at follow up $(\mathrm{p}>0.05)$.

\begin{tabular}{ll}
$\begin{array}{l}\text { Abstract P138 Table } 1 \\
\text { discharges }\end{array}$ & Employment status of COVID-19 \\
\hline $\mathrm{N}$ & 138 \\
\hline Essential services & $41(30)$ \\
Office/admin & $37(27)$ \\
Healthcare & $16(12)$ \\
Non essential services & $15(11)$ \\
Public transport & $10(7)$ \\
Enforcement & $4(3)$ \\
Heavy Goods Vehicle driver & $3(2)$ \\
Carers & $2(1)$ \\
Unknown & $10(7)$
\end{tabular}

Conclusion Our preliminary data suggests significant symptom burden within 6 weeks post discharge after a COVID-19 infection admission, which may impact on the ability of patients to return to work. In the present analysis there was no significant interaction between return-to-work status and covid severity.

\section{P139 THE SYMPTOMATOLOGY OF LONG COVID PATIENTS IN CHESHIRE AND MERSEYSIDE}

S Kimyongur, G Heppenstall-Harris, A Barnes, A Flatt, E Bruchez, M Stolbrink, N Nwosu, L Watkins, G Tack. Liverpool University Hospitals NHS Foundation Trust, Liverpool, UK

\subsection{6/thorax-2021-BTSabstracts.248}

Background A growing proportion of patients experience ongoing symptoms for more than 12 weeks following an initial COVID-19 illness. Long COVID or post-COVID syndrome is an increasing burden to the NHS. NHS England has mandated the setup of regional long COVID clinics to manage this demand. ${ }^{1}$ There is very little understanding of the range of symptoms and the symptom burden to patients.

Aims We describe self-reported symptoms and compare breathlessness and fatigue scoring for patients referred to the Cheshire and Merseyside Long COVID assessment hub, which serves a population of $>2.5$ million, between February 2021 and April 2021.

Methods Retrospective review of case notes of patients assessed in our virtual Long COVID assessment hub. Data was analysed using paired t-tests. Fatigue was scored on a scale of 0 (no fatigue) to 10 (extreme fatigue). Breathlessness was assessed using Borg breathlessness scale and MRC dyspnoea score.

Results 332 patients were assessed, with the following symptoms described in table 1 . We found a significant increase in self-reported Borg breathlessness (4.9 vs 0.2, p<0.0001), MRC dyspnoea $(2.5$ vs $1.1, \mathrm{p}<0.0001)$ and fatigue $(7.1$ vs $0.7, \mathrm{p}<0.0001)$ at time of assessment, when compared with reported pre-morbid state.

Discussion Patients experience significant worsening breathlessness and fatigue. This significant symptom burden emphasises the need for dedicated Long COVID assessment hubs to support patients. Further research should investigate whether pulmonary rehabilitation and dedicated psychological input can improve patients' symptoms.

\section{REFERENCE}

1. NHS England and NHS Improvement (2021). Long COVID: the NHS plan for $2021 / 22$.

Abstract P139 Table 1 Percentage of patients reporting various symptoms of Long COVID

\begin{tabular}{ll}
\hline Symptom & Percentage of patients \\
\hline Fatigue & $98 \%$ \\
Worsening breathlessness & $97 \%$ \\
Cognitive symptoms & $91 \%$ \\
Myalgia & $66 \%$ \\
Chest pain & $47 \%$ \\
Ongoing cough & $46 \%$ \\
Anosmia & $45 \%$ \\
\hline
\end{tabular}

